\title{
Heel pad avulsion injury: an approach with hyperbaric oxygen therapy
}

\author{
Pradeoth Korambayil Mukundan, Prashanth Varkey Ambookan \\ Department of Plastic Surgery and Burns, Jubilee Institute of Surgery for Hand, Aesthetic and Microsurgery, Jubilee Mission Medical \\ College and Research Institute, Thrissur 680007, Kerala, India.
}

Address for correspondence: Dr. Pradeoth Korambayil Mukundan, Flat No. 102, Nandana, Haritha Gardens, Vadookara, Thrissur 680007, Kerala, India. E-mail: pradeoth@gmail.com

\begin{abstract}
Aim: Crush injuries of the foot are often associated with partial or complete degloving of the heel pad. The purpose of this study is to present an algorithm for the management of various types of heel pad avulsion injuries, including hyperbaric oxygen (HBO) therapy in the treatment regimen. Methods: We present a prospective study of 27 patients with various types of heel pad avulsion managed in our institution from December 2012 to June 2013. Heel pad avulsion injuries were classified according to the angiosomal pattern. Partial or complete avulsions were classified and treated accordingly. HBO therapy was administered postoperatively. The postoperative period, hospital course, and follow-up were documented in patients with heel pad avulsion injuries. Results: Of 27 patients, 20 cases presented with partial avulsion and 7 cases were complete avulsion. Of 20 cases of partial avulsion, one of the flaps was anchored with K-wire. Nineteen cases of partial heel pad avulsion were managed by suturing. Eight patients out of 20 required skin grafting as a secondary procedure at a later date. Out of 7 cases of complete avulsion, one was managed by full-thickness skin grafting, one case by reverse sural artery flap coverage, and four cases were managed by free tissue transfer. No flap revisions were required, and no complications were experienced for the transferred flaps. Conclusion: HBO therapy may be a useful adjunct in the treatment of heel pad avulsion injuries.
\end{abstract}

Key words:

Angiosome, heel pad avulsion, hyperbaric oxygen therapy, soft tissue reconstruction

\section{INTRODUCTION}

Foot trauma is a significant cause of morbidity among the working age population. Crush injuries to the foot are often associated with avulsion injuries of the heel pad. Heel pad avulsion injuries are always a challenge for the plastic surgeons, as the reconstructed tissue, even though sensate, may not match the unique and complex nature of the native fat pad structure. Heel pad avulsion injuries may be partial or complete. ${ }^{[1]}$ When the avulsed heel pad tissue is avascular or clinically nonviable, avulsion is said

\begin{tabular}{|l|l|}
\hline \multicolumn{2}{|c|}{ Access this article online } \\
\hline Quick Response Code: & Website: \\
\hline & www.parjournal.net \\
\hline & \\
\hline & \\
\hline
\end{tabular}

to be complete and may require removal of the avascular tissue and reconstruction with local or distant flaps. Providing sensate and glabrous skin may not be possible in patients with extensive injuries. ${ }^{[2]}$ There are clinical situations in which the avulsed heel pad structure may be viable, requiring debridement and anchoring of the heel pad flap in position. In cases of partial avulsion, the heel pad is, usually, debrided, reattached in position by sutures or K-wire fixation, and further surgical interventions are postponed until there has been a demarcation of the nonviable tissue. ${ }^{[1]}$ As the heel pad tissue is irreplaceable, there is a need for alternative methods of preserving the marginal tissue as well as preventing ischemia and hypoxic advancement of the injured tissue. In this prospective study, various treatment modalities including hyperbaric oxygen (HBO) therapy, primary closure, full thickness skin grafting/split thickness skin grafting (FTSG/STSG), and local or distant flap coverage were utilized to preserve and reconstruct the valuable heel pad tissue. 


\section{METHODS}

Twenty-seven patients with heel pad avulsion (isolated/ combined) were treated over a period of 7 months (December 2012 to June 2013). The avulsed heel pad flaps were classified on the basis of the angiosomal concept. Heel pad flaps based distally were classified as tissue receiving its vascular supply from the medial plantar and lateral plantar artery angiosomal territories. Flaps based distally and medially were considered to receive their vascular supply from the medial and lateral plantar arteries and the calcaneal branch of the posterior tibial artery. Flaps based distally and laterally were considered to have a vascular supply based on the medial and lateral plantar arteries and the calcaneal branch of the peroneal artery. Those flaps with proximal continuity were classified as having their blood supply from perforators of either the posterior tibial or peroneal vessels.

Following initial assessment and resuscitation of the patient according to the ATLS protocols, acutely presenting heel pad avulsion injuries were assessed for the extent of degloving, skeletal injury, associated soft tissue loss, vascularity to the heel pad flap, and flap avulsion patterns. Patients were informed about the line of management, possible treatment modalities, and the need for additional surgery procedures pertaining the pattern of injuries. Patients were started on empirical antibiotic therapy, including a third-generation cephalosporin and anaerobic coverage. Adequate analgesia was assured. Thorough wound debridement and fixation of the fractures was performed. In cases of partial avulsion requiring the anchorage, HBO therapy was initiated during the immediate postoperative period. Six sessions of HBO therapy, each session lasting $1 \mathrm{~h}$ and continued for 6 days, was administered to the patients postoperatively. In cases of complete avulsion, the nonviable tissues were debrided, and depending upon the patient's condition, soft tissue reconstruction was performed as early as possible. Postoperatively, 6 HBO therapy sessions were administered for all patients. Immobilization of the limb was done in all cases. Outcomes following each type of management and secondary procedures performed were noted.

The review board of Jubilee Mission Medical College and Research Institute approved this study.

\section{RESULTS}

The mode of injury for all patients was road traffic accident. Out of 27 patients, there were 5 female (18.1\%) and 22 male patients $(81.9 \%)$. Mean age was 34.18 years (range: 5-53 years). For 12 patients the avulsed flap was based distally, in 7 patients the flap was based distally and laterally, in 5 patients the flap was based distally and medially, in 2 patients the flap had proximal and distal connections with disruption in the medial and lateral aspects and in 1 patient the flap was continuous only in the lateral and medial aspects. Out of 20 cases of partial avulsion, one of the flaps was anchored with K-wires by the orthopedic department, and then referred to plastic surgery for soft tissue coverage. Nineteen cases of partial heel pad avulsion were managed by primary closure. Eight patients out of 20 required skin grafting as a secondary procedure at a later date. Out of 7 cases of complete avulsion, one was managed by full-thickness skin grafting, one case by reverse sural artery flap coverage, and four cases were managed by free tissue transfer. Among the free tissue transfer, two were latissimus dorsi muscle flaps, one was a gracilis muscle flap, and one was an anterolateral thigh flap [Table 1]. There were no complications following flap transfer and graft take was adequate. The patients in our series did not experience the common side-effects of HBO therapy such aural or pulmonary barotrauma or transient reversible myopia during the treatment sessions. No complications were noted during the follow-up period.

\section{Case I}

A 43-year-old female was admitted with a crush injury to the right leg and foot region following a crush injury by a heavy vehicle [Figure 1a]. The patient presented with soft tissue loss over the anterior aspect of the leg and dorsum of the foot. The heel pad was avulsed from the calcaneum but was continuous to the proximal and the distal aspect by the skin and subcutaneous tissue [Figure 1b]. Stabilization of the ankle and heel pad was performed with an external fixator following wound debridement. Soft tissue coverage of the anterior aspect of the leg and dorsum of the foot was provided by a latissimus dorsi free flap and split-thickness skin grafting [Figure 1c and d]. The vessels of the latissimus dorsi flap were anastomosed end-to-side to the posterior tibial vessels as the anterior tibial vessels were avulsed up to the level of middle third of the leg. HBO therapy was administered in 6 sessions postoperatively. Following demarcation of the avascular tissue over the medial part of the leg and proximal heel pad, nonviable tissue was debrided. Because there was adequate soft tissue padding over the calcaneum, skin grafting was performed [Figure 1e-g]. Six sessions of HBO therapy were administered following skin grafting.

\section{Case 2}

An 18-year-old male was admitted with a crush injury of the right leg and foot with heel pad avulsion. Skin and subcutaneous tissue were connected in the proximal and distal aspects [Figure 2a]. The heel pad was anchored with K-wires by the orthopedic department, and the patient was then referred to plastic surgery for further management [Figure 2b]. HBO therapy was administered for six sessions. Following demarcation of the nonviable tissue, the avascular tissue was debrided [Figure $2 \mathrm{c}$ and $\mathrm{d}$ ]. Most of the foot pad tissue was found to be preserved, and skin grafting was sufficient for coverage of the soft tissue defect following debridement [Figure 2e and f]. Six additional sessions of HBO therapy were administered following skin grafting.

\section{Case 3}

A 52-year-old male was admitted with heel pad avulsion based distally [Figure 3a]. Primary closure was performed following debridement [Figure $3 \mathrm{~b}$ and $\mathrm{c}$. HBO therapy was 
administered for 6 sessions. The wound healed without any additional intervention [Figure 3d].

\section{DISCUSSION}

Crush injuries to the foot are a significant cause of morbidity among the working age population and are commonly associated with degloving of the heel pad.[1] The heel pad flaps are typically elevated in a posterior to anterior direction. Crush injuries may disrupt the heel pad in various flap patterns, which are medially based, laterally based, medially and anteriorly based, or laterally and anteriorly based. In some cases, the soft tissues in the medial, posterior and lateral areas are disrupted, leaving

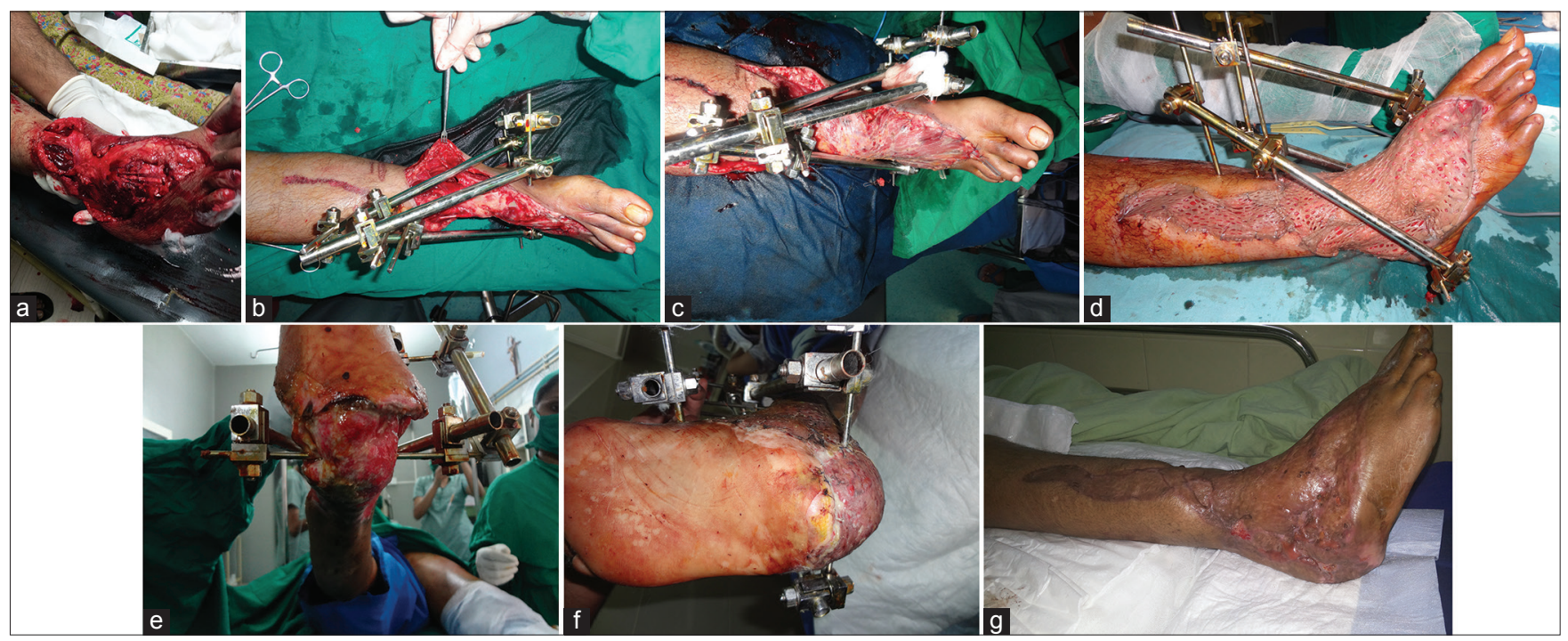

Figure 1: (a) Crush injury to the right leg and foot region; (b) soft tissue loss anterior aspect of leg and dorsum of foot with heel pad avulsed from the calcaneum with continuity to the proximal and the distal part; (c) soft tissue coverage of leg and dorsum of foot with latissimus dorsi free tissue transfer; (d) free muscle tissue covered with split thickness skin graft; (e) status of heel region following debridement and hyperbaric oxygen therapy after 3 weeks of injury; (f) postoperative picture following skin grafting over heel pad region; (g) late postoperative picture

Table 1: The patients treated for heel pad avulsion injury

\begin{tabular}{|c|c|c|c|c|c|}
\hline No. & $\begin{array}{l}\text { Age (years)/ } \\
\text { gender }\end{array}$ & $\begin{array}{l}\text { Mode of } \\
\text { injury }\end{array}$ & $\begin{array}{l}\text { Base of } \\
\text { vascularity }\end{array}$ & $\begin{array}{l}\text { Primary management } \\
\text { following debridement }\end{array}$ & $\begin{array}{l}\text { Secondary } \\
\text { procedure }\end{array}$ \\
\hline 1 & $32 /$ male & RTA & Distal & Primary suturing & \\
\hline 2 & 23/male & RTA & Distal & Primary suturing & \\
\hline 3 & 24/female & RTA & Distal & Primary suturing & SSG \\
\hline 4 & 42/male & RTA & Distal & Primary suturing & SSG \\
\hline 5 & $53 /$ male & RTA & Distal & Latissimudorsi flap & \\
\hline 6 & 34/female & RTA & Distal & Latissimus dorsi flap & \\
\hline 7 & 42/male & RTA & Distal and lateral & Primary suturing & \\
\hline 8 & $27 /$ male & RTA & Distal and medial & Primary suturing & \\
\hline 9 & $34 /$ male & RTA & Distal & Gracilis flap & \\
\hline 10 & $5 /$ male & RTA & Distal & FTSG & \\
\hline 11 & 52/female & RTA & Distal & Primary suturing & \\
\hline 12 & 25/male & RTA & Distal and lateral & Primary suturing & SSG \\
\hline 13 & $27 /$ male & RTA & Distal and lateral & Primary suturing & \\
\hline 14 & $21 /$ male & RTA & Distal and lateral & Primary suturing & SSG \\
\hline 15 & 28/male & RTA & Distal and lateral & Primary suturing & \\
\hline 16 & 43/male & RTA & Distal and lateral & Primary suturing & \\
\hline 17 & $42 /$ male & RTA & Lateral and medial & Primary suturing & SSG \\
\hline 18 & $37 /$ male & RTA & Distal and lateral & Primary suturing & \\
\hline 19 & 43/female & RTA & Proximal and distal & Latissimus dorsi flap & SSG \\
\hline 20 & $35 /$ male & RTA & Distal and medial & Primary suturing & \\
\hline 21 & 39/male & RTA & Distal and medial & Primary suturing & \\
\hline 22 & 42/male & RTA & Distal and medial & Primary suturing & \\
\hline 23 & 45/male & RTA & Distal and medial & Primary suturing & SSG \\
\hline 24 & 21/female & RTA & Distal & Primary suturing & SSG \\
\hline 25 & 18/male & RTA & Proximal and distal & Anchorage with K-wire & SSG \\
\hline 26 & 47/male & RTA & Distal & Reverse sural flap & \\
\hline 27 & 42/male & RTA & Distal & Anterolateral thigh flap & \\
\hline
\end{tabular}

RTA: Road traffic accident, FTSG: Full thickness skin grafting, SSG: Split thickness skin grafting 


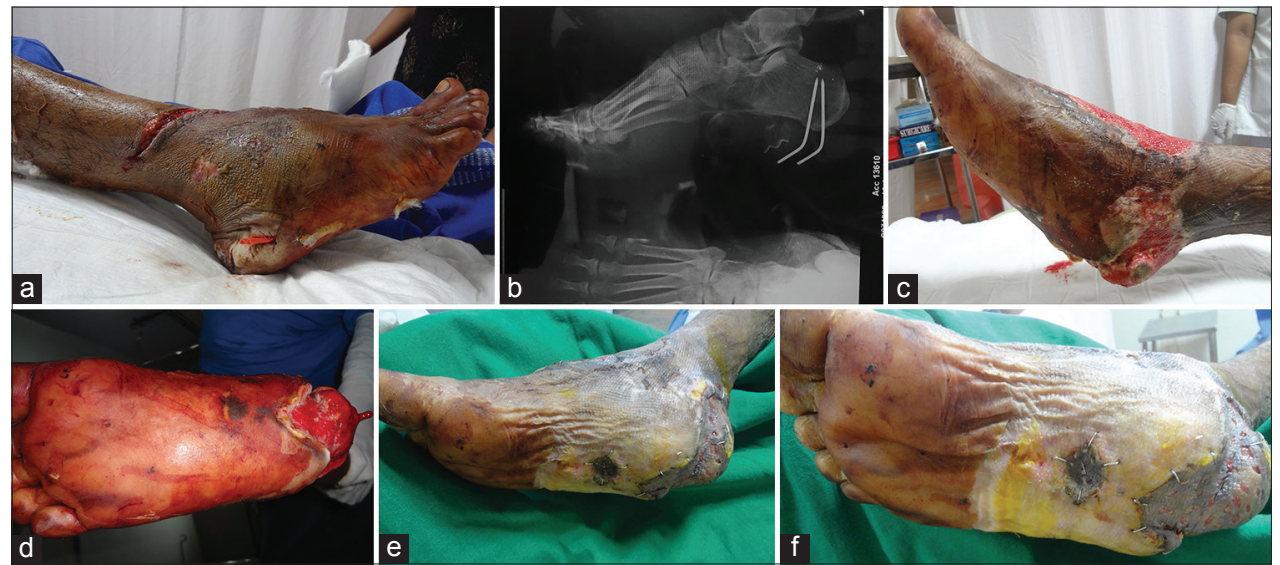

Figure 2: (a) Crush injury right leg and foot with heel pad avulsed skin and subcutaneous tissue connectivity in the proximal and distal part; (b) X-ray lateral view right foot showing K-wires used for stabilization of avulsed heel pad; (c) appearance of foot 3 weeks following debridement and hyperbaric oxygen treatment session; (d) plantar view of foot following 3 weeks; (e) medial view of the foot following skin grafting; (f) plantar view of the foot following skin grafting

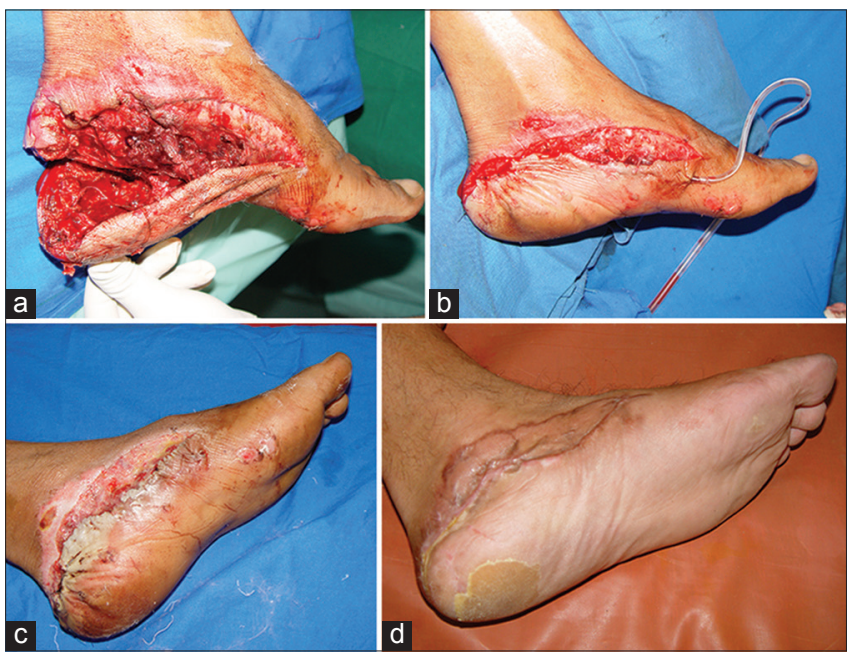

Figure 3: (a) Heel pad avulsion based distally-medial view of left foot foot; (b) wound debridement and primary suturing after placing a suction drain; (c) picture presenting the wound status during immediate postoperative period; (d) late postoperative picture

only a soft tissue bridge anteriorly. In some instances, the heel pad tissue is degloved in a "bucket handle" configuration over the calcaneal bone, with continuity proximally and distally and disruption of the soft tissue in the medial and lateral aspects [Figure 4]. The heel pad tissue, with its unique architecture, demands preservation in cases of any possible vascular supply. Premature efforts to remove doubtful tissue may result in loss of valuable sensate heel pad tissue, which might have been preserved by delayed intervention. However, a delayed intervention may also result in decreased survival rates of local or distant tissue transfer secondary to tissue edema and an increase in inflammatory factors. Since the zone of soft tissue compromise may extend beyond the zone of injury of the foot, reconstruction of heel pad with local or distant soft tissue reconstruction can be challenging. In addition, efforts to replace the heel pad with vascularized or regional flaps may be limited by decreased fine sensation, bulky soft tissues, and alteration in gait function. ${ }^{[3]}$

The vascular anatomy of the leg and foot by means of the angiosomal concept provides us with a better approach for

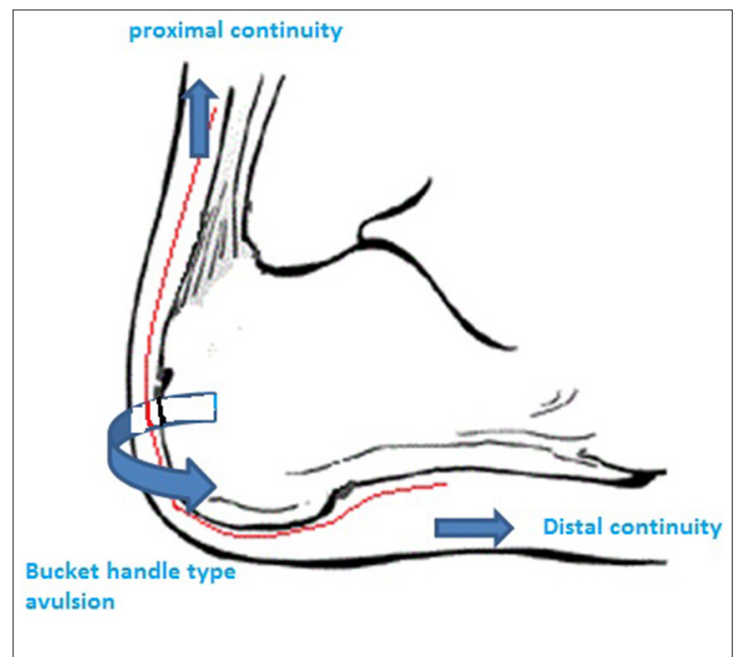

Figure 4: Diagram illustrating heel pad tissue degloved as a bucket handle type over the calcaneal bone with continuity proximally and distally with disruption of soft tissue medial and lateral aspect

the management of such complicated injuries [Figure 5]. Out of 6 angiosomes of the foot and ankle, 3 angiosomes are supplied by the posterior tibial artery, 2 angiosomes are supplied by the peroneal artery, and 1 angiosome is supplied by the anterior tibial artery..$^{[4]}$ The calcaneal branch of the posterior tibial artery supplies the medial aspect of the ankle and the plantar aspect of the heel pad region. The medial plantar branch of the posterior tibial artery feeds the medial aspect of the plantar instep. The lateral plantar branch of the posterior tibial artery feeds the lateral aspect of the forefoot, the plantar aspect of the midfoot, and the plantar aspect of the forefoot. The calcaneal branch of the peroneal artery supplies the lateral aspect of the heel pad region. ${ }^{[5]}$ Taylor et al. ${ }^{[4]}$ found that these angiosomes are interconnected by either reduced caliber choke vessels or by anastomotic arteries. The principle of utilizing HBO therapy for ulcers with vascular insufficiency and in radiation-induced wounds can be applied to the salvage of vital heel pad tissue. HBO therapy creates an increase in dissolved oxygen in the plasma where there is increased partial pressure of arterial oxygen. Oxygen delivery through 


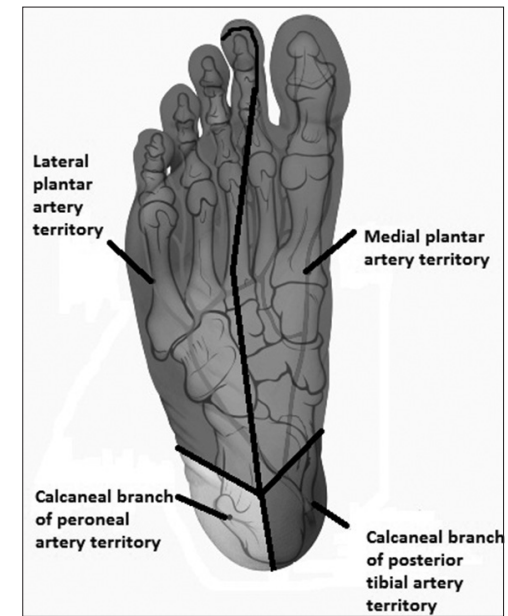

Figure 5: Angiosomal pattern of the heel pad and plantar foot region

the plasma is increased through hemoglobin-bound oxygen, facilitating oxygenation of the deprived tissue, stimulating angiogenesis, fibroblast proliferation, leukocyte oxidative killing, antibiotic synergy, toxin inhibition and vasoconstriction. Vasoconstriction significantly reduces tissue edema without hindering oxygenation. Decreasing edema is desirable in complicated plastic surgical wounds to relieve pressure on the surrounding vessels and structures. Oxygen delivery also leads to mature collagen formation and stimulates angiogenesis. ${ }^{[6]}$ Ulkuir et al..$^{[7]}$ illustrated the usefulness of HBO treatment during the delay period of the flap which can both decrease the time period needed for the delay procedure and increased the effect of the flap delay. The current study illustrates the utility of hyperbaric therapy in the treatment of heel pad avulsion injuries. Most heel pad avulsion injuries with partial continuity act similar to a delayed flap. Hence, considering the valuable heel pad tissue and the need for its preservation at any cost, management should include a modality which can potentially enhance vascularity and wound healing potential during the period of demarcation of necrotic tissue.

Hyperbaric oxygen therapy utilizes $100 \%$ oxygen at pressures greater than atmospheric pressure. In the current study, HBO was administered in a Monoplace chamber in which a single patient is placed in a chamber that is then pressurized with $100 \%$ oxygen. The pressure inside the chamber is adjusted, ranging from 2.0 ATA to 2.4 ATA for duration of $90 \mathrm{~min}$. As an adjunct to surgery and antibiotics, HBO therapy can significantly decrease costs and complications. ${ }^{[8]}$ Vasoconstriction reduces edema and tissue swelling while ensuring adequate oxygen delivery and is thus useful in acute trauma wounds. Hyperoxygenation of the crush injury and compartment syndrome followed by flap salvage results in immune stimulation by restoring white blood cell function and enhancing phagocytic capabilities. Neo-vascularization in hypoxic areas is augmented by fibroblastic activity and capillary growth. ${ }^{[8]}$ Adequate shock management, direct surgical intervention with debridement, repair of soft tissues and any damaged vessels, and stabilization of bony elements are of paramount importance. Adjuvant HBO can be given early to prevent large regions of ischemic necrosis, minimize the frequency and extent of tissue necrosis, reduce edema, control infection, support healing, and prevent reperfusion injury. ${ }^{[9]}$

At the authors' institution, a strategy has been developed to overcome these difficulties and to successfully manage these patients with a combined approach that maximizes tissue perfusion and oxygenation, allowing for surgical correction of such injuries. The current treatment algorithm [Figure 6] begins with surgical debridement and initiation of HBO therapy in the immediate postoperative period. HBO provides supersaturation of the plasma with oxygen, allowing a several-fold increase in the oxygen diffusion gradient. Combination of the modalities allows preservation of marginal tissue, prevention of advancing ischemia and hypoxia and maximum preservation of heel pad tissue. This approach has been used in the current series of 27 patients, achieving maximal preservation of the heel pad with a return to ambulation.

Out of 27 patients, the avulsed flap was distally-based in 12 patients, distally and laterally based in 7 patients and distally and medially based in 5 patients. In 2 patients the flap had proximal and distal connections with disruption in the medial and lateral aspects, and in 1 patient the flap was continuous in only the lateral and medial aspects. In all cases, initial wound debridement was carried out and HBO therapy sessions were started. If flap coverage was required, surgery was scheduled as soon as the patient was stable. HBO therapy was withheld on the day of surgery and recommenced on postoperative day one. When skin grafting was performed at a later date, surgery was followed by six sessions of HBO therapy. Out of 20 cases of partial avulsion, one flap was anchored with a K-wire from the orthopedic department, and then referred to plastic surgery for soft tissue management. Nineteen cases of partial heel pad avulsion were closed primarily. Eight patients out of 20 required skin grafting as a secondary procedure at a later date. Split-thickness skin grafting was the method of choice when adequate soft tissue padding was present. In the authors' experience, the risk of calcaneal bone exposure was decreased in patients treated with HBO therapy. Split thickness grafting can suffice if adequate soft tissue padding is present. ${ }^{[2]}$ However, there is a need for a randomized controlled study of the preservation of heel fat pad tissue following HBO therapy. Out of 7 cases of complete avulsion, 1 patient was managed by full-thickness skin grafting. The benefit of HBO therapy is greatest in cases in which relatively large areas of tissue are grafted, as in full-thickness skin grafting. ${ }^{[8]}$ One patient with a heel pad defect was treated by reverse sural artery flap coverage, and four patients were managed by free tissue transfer. Among the free tissue transfer cases, 2 were latissimus dorsi muscle flaps, 1 was a gracilis muscle flap and one was an anterolateral thigh flap. There were no complications following any flap transfers. The patients in the current series did not experience the common side effects of HBO therapy, including aural or pulmonary barotrauma and transient reversible myopia during the treatment session. No recent literature is available on management of heel pad avulsion injuries using surgery and HBO therapy as a combined modality. Other advanced wound care 
modalities including negative pressure wound therapy and limited-access dressings could well be utilized along with HBO therapy for the management of such challenging injuries. Complications and problems in wound healing are bound to occur in crush injuries of the foot involving heel pad region. HBO therapy is an adjunct in the management of marginal tissues, but cannot be utilized in an avascular environment which requires surgical intervention. Measures that could improve the outcome with the adjunct use of HBO therapy should be considered [Figure 7].

In conclusion, the goal of management of heel pad avulsion injuries is to preserve as much viable heel pad tissue as possible and to provide sensate, stable coverage. Principles of wound management with wound evaluation,

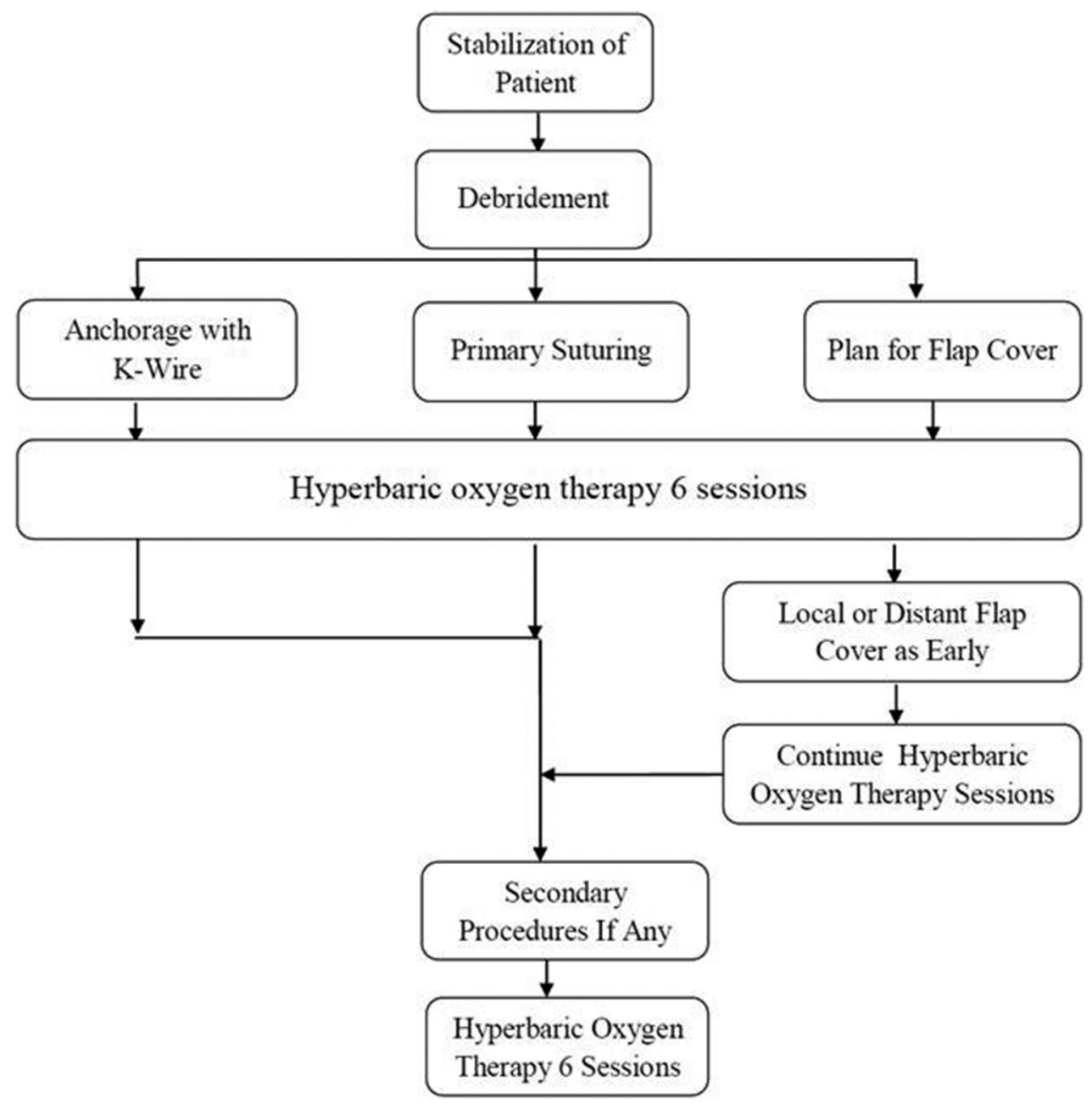

Figure 6: Algorithm for management for heel pad avulsion injuries in our institution

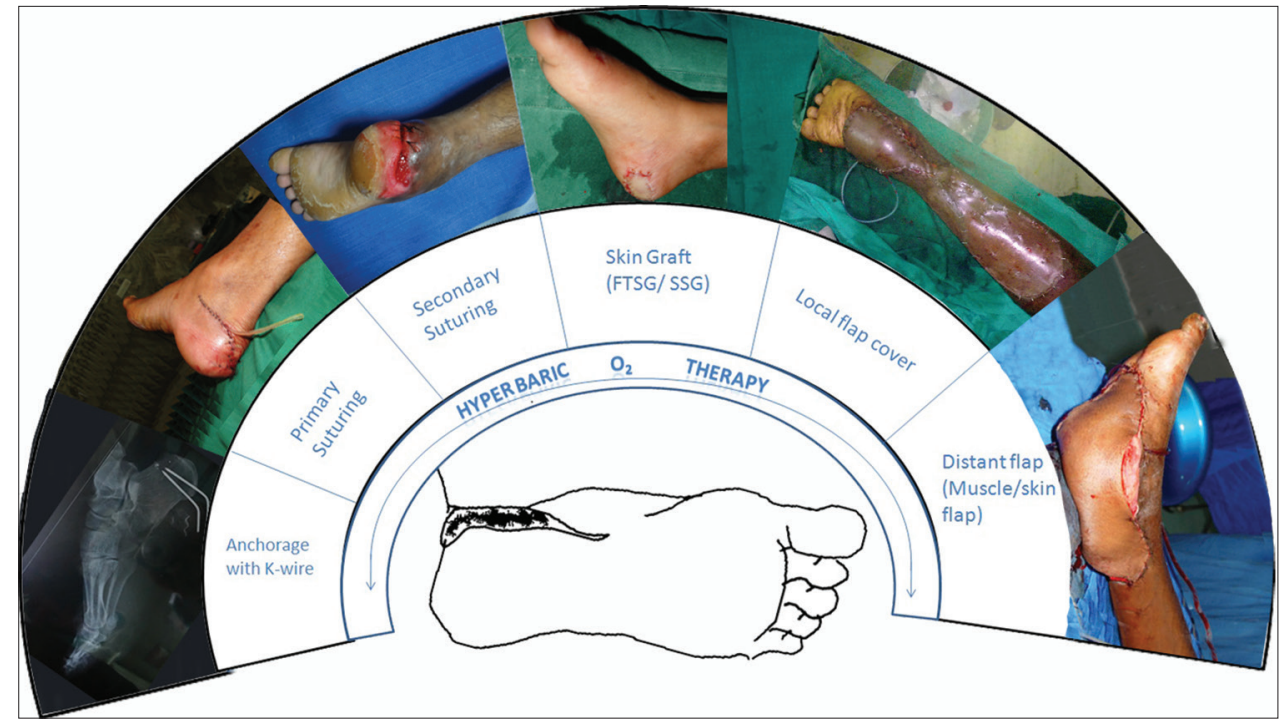

Figure 7: Hyperbaric oxygen therapy as an adjunct in the management of various patterns of heel pad avulsion injuries 
debridement with preservation of viable tissue, fracture reduction, prevention of infection, and adequate soft tissue reconstruction are important in the management of heel pad avulsion injuries. The simplest and most appropriate technique is likely to produce the best outcome. This study presents a simple management algorithm for the management of various types of heel pad avulsion injuries. HBO therapy may be used as a useful adjunct in the treatment of such complicated injuries to offer speedy recovery as well as cost benefits.

\section{REFERENCES}

I. Mohammed R, Metikala S. Anchorage of partial avulsion of the heel pad with use of multiple kirschner wires. A report of four cases. JBJS Case Connect 20I2;2:20.

2. Suri MP, Patel AG, Vora HJ, Raibagkar SC, Mehta DR, Vyas UH. Post-traumatic posterior heel soft tissue defect reconstruction. Indian J Plast Surg 2005;38: 138-43.

3. Levin LS. Foot and ankle soft-tissue deficiencies: who needs a flap? Am J Orthop (Belle Mead NJ) 2006;35: I I-9.

4. Acín F, Varela C, López de Maturana I, de Haro J, Bleda S, Rodriguez-Padilla J.
Results of infrapopliteal endovascular procedures performed in diabetic patients with critical limb ischemia and tissue loss from the perspective of an angiosome-oriented revascularization strategy. Int J Vasc Med 20I4;20I4:270539.

5. Attinger CE, Evans KK, Bulan E, Blume P, Cooper P. Angiosomes of the foot and ankle and clinical implications for limb salvage: reconstruction, incisions, and revascularization. Plast Reconstr Surg 2006; I 17:S26I-93.

6. Latham E, Hare MA, Neumeister M. Hyperbaric oxygen therapy. eMedicine. In: Schraga ED, Windle ML, Scudder L, editors. Medscape. Available from: http://www.emedicine.com/plastic/topic526.htm. [Last accessed on Aug 2014].

7. Ulkür E, Karagoz H, Ergun O, Celikoz B, Yildiz S, Yildirim S. The effect of hyperbaric oxygen therapy on the delay procedure. Plast Reconstr Surg 2007; I 19:86-94.

8. Bhutani S, Vishwanath G. Hyperbaric oxygen and wound healing. Indian J Plast Surg 2012;45:316-24.

9. Kemmer A. Crush injury and other traumatic ischemia. In: Mathieu D, editor. Handbook on Hyperbaric Medicine. Netherlands: Springer; 2006. p. 3II-2.

How to cite this article: Mukundan PK, Ambookan PV. Heel pad avulsion injury: an approach with hyperbaric oxygen therapy. Plast Aesthet Res 2015;2:56-62.

Source of Support: Nil, Conflict of Interest: None declared.

Received: 19-08-2014; Accepted: 28-09-2014 